\title{
La gamificación a través de plataformas E-learning: Análisis cienciométrico de una pedagogía emergente implantada mediante de las TIC
}

Álvaro Cobos Sánchez - Universidad de Granada
Juan José Padial Suárez - Universidad de Granada
Emilio Berrocal de Luna - Universidad de Granada
0000-0001-8982-4468

D $0000-0003-1960-363 X$

(D) $0000-0002-3562-8135$

Recepción: 11.07.2021 | Aceptado: 16.10.2021

Correspondencia a través de ORCID: Álvaro Cobos Sánchez

iD 0000-0001-8982-44687

Citar: Cobos Sánchez, A, Padial Suárez, JJ y Berrocal de Luna, E (2021). La gamificación a través de plataformas E-learning: Análisis cienciométrico de una pedagogía emergente implantada mediante de las TIC. REIDOCREA, 10(30), 1-20.

Resumen: La digitalización de la sociedad ha generado un avance acelerado a todos los niveles. En el caso de la educación, se ha creado un nuevo ecosistema didáctico alternativo a la modalidad tradicional, llamado e-learning. Está modalidad presenta una serie de diferencias con respecto a la modalidad tradicional que ofrece una serie de ventajas para que parte de la ciudadanía siga formándose. Una forma de aplicar el e-learning es a través de una plataforma virtual. Asimismo, debido a la crisis sanitaria se ha puesto en el foco a dicha modalidad, evaluando sus beneficios y sus inconvenientes. Algunos de sus inconvenientes, como la falta de un componente motivacional, o la falta de compromiso, pueden paliarse a través de la implementación de la gamificación en este tipo de modalidad. Este estudio se realiza para profundizar en el funcionamiento de la combinación del e-learning y la gamificación. Mediante un estudio cienciométrico se analiza una muestra de 210 producciones científicas indexadas en la base de datos Web of Science, examinando principalmente, la producción diacrónica, los autores más citados y productivos, las instituciones y departamentos más productivos. Destaca la tendencia exponencial de publicación sobre el fenómeno en cuestión, además del impacto de autores y producciones científicas que se centran en describir las limitaciones de una gamificación centrada en la motivación extrínseca, y la necesidad de apoyarse en una motivación más intrínseca para que la gamificación se aplique de manera óptima.

Palabra clave: Gamificación

Gamification through E-learning platforms: Scientometric analysis of an emerging pedagogy used through ICT

Abstract: The digitization of society has generated accelerated progress at all levels. In the case of education, a new alternative didactic ecosystem to the traditional modality, called e-learning, has been created. This modality presents a series of differences with respect to the traditional modality that offers a series of advantages so that part of the citizenship continues to be trained. One way to apply e-learning is through a virtual platform. Likewise, due to the health crisis, the focus has been on this modality, evaluating its benefits and drawbacks. Some of its drawbacks, such as the lack of a motivational component, or the lack of commitment, can be alleviated through the implementation of gamification in this type of modality. This study is carried out to deepen the operation of the combination of e-learning and gamification. Through a scientometric study, a sample of 210 scientific productions indexed in the Web of Science database is analyzed, examining mainly diachronic production, the most cited and most productive authors, the most productive institutions and departments, among others. The exponential trend of publication on the phenomenon in question stands out, in addition to the impact of authors and scientific productions that focus on describing the limitations of a gamification focused on extrinsic motivation, and the need to rely on a more intrinsic motivation for gamification applied more optimally.

Keyword: gamification

\section{Introducción}

\section{E-learning}

Algo que caracteriza el acelerado avance tecnológico de las sociedades actuales ha sido el uso de las tecnologías de la información y la comunicación (TIC), como, por ejemplo, internet, y puesto que el uso de las Tics ha revolucionado el mundo a todos los 
niveles, la educación no iba a ser menos. La educación, en todos los niveles, gracias a la digitalización que han aportado las Tics, ha conseguido integrar un nuevo ecosistema didáctico con un proceso de enseñanza-aprendizaje diferente, llamado e-learning (Valverde-Berrocoso et al., 2020). Se puede definir el e-learning como un sistema de información y comunicación basado en el uso de internet, el cual a través de herramientas digitales y distintos materiales educativos pretende crear un entorno de aprendizaje adaptado al individuo, abierto e interactivo, y centrando el foco en el alumno con la finalidad de conseguir un proceso de aprendizaje adecuado (Rodrigues et al., 2019). Además, el e-learning tiene implícito una serie de características que lo diferencian significativamente de las modalidades más tradicionales. García-Aretio (2020) enumeró que principalmente hay cuatro características que lo diferencian: La separación geográfica del docente y los alumnos; La autonomía en el proceso de aprendizaje del alumnado; Comunicación sincrónica y asincrónica entre los compañeros a través de las herramientas disponibles; y el apoyo por parte de una institución. El elearning puede subdividirse principalmente en 2 tipos, el e-learning autónomo dirigido por el propio estudiante, donde incluimos los MOOC (masive online open course) y el elearning dirigido por un tutor (Velazco Flórez et al., 2017).

\section{Bases epistemológicas de la modalidad e-learning}

Teniendo en cuenta las diferencias entre la modalidad e-learning y las modalidades más tradicionales, es necesario saber si el proceso de enseñanza-aprendizaje presenta matices en función de la modalidad aplicada. Kibuku y Ochieng, (2018) realizaron una investigación donde evaluaron la aplicación de las principales teorías del aprendizaje a la modalidad e-learning, destacando los elementos que principalmente aportan a la explicación del proceso de enseñanza-aprendizaje, y resaltando sus limitaciones y/o deficiencias a la hora de aplicarlas a un aprendizaje virtual o e-learning. En la siguiente tabla pueden apreciarse las contribuciones y limitaciones que presentan las principales teorías del aprendizaje aplicadas a la modalidad e-learning:

\section{Tabla 1}

Síntesis sobre los aciertos y limitaciones de las Teorías clásicas de aprendizaje sobre el E-learning a partir de Kibuku y Ochieng, (2018)

\begin{tabular}{|c|c|c|}
\hline $\begin{array}{l}\text { Teorías clásicas } \\
\text { aplicadas al } \\
\text { aprendizaje }\end{array}$ & $\begin{array}{c}\text { Aciertos y/contribuciones de la } \\
\text { teoría al E-learning }\end{array}$ & $\begin{array}{l}\text { Limitaciones y críticas a la } \\
\text { teoría con respecto al E- } \\
\text { learning }\end{array}$ \\
\hline Conductismo & $\begin{array}{l}\text { - Se apoya en el comportamiento } \\
\text { observable y medible como } \\
\text { indicador del aprendizaje. } \\
\text { - Permite plantear pasos de } \\
\text { instrucción secuenciados. } \\
\text { - Se centra en un diseño del } \\
\text { contenido del aprendizaje de } \\
\text { manera estructurada. } \\
\text { - Es apropiada para procesos de } \\
\text { enseñanza-aprendizaje } \\
\text { sencillos, antes de pasar a los } \\
\text { más complejos. }\end{array}$ & $\begin{array}{l}\text { - No es posible observar } \\
\text { todos los } \\
\text { comportamientos } \\
\text { implicados, lo cual alienta } \\
\text { a los alumnos a centrarse } \\
\text { en aprender a hacer y } \\
\text { dejar de lado el aprender } \\
\text { a ser. } \\
\text { - El tutor tiene un rol } \\
\text { protagonista mientras que } \\
\text { el alumnado tiene un } \\
\text { papel totalmente pasivo } \\
\text { en el proceso. } \\
\text { - Falta de un componente } \\
\text { social. }\end{array}$ \\
\hline
\end{tabular}




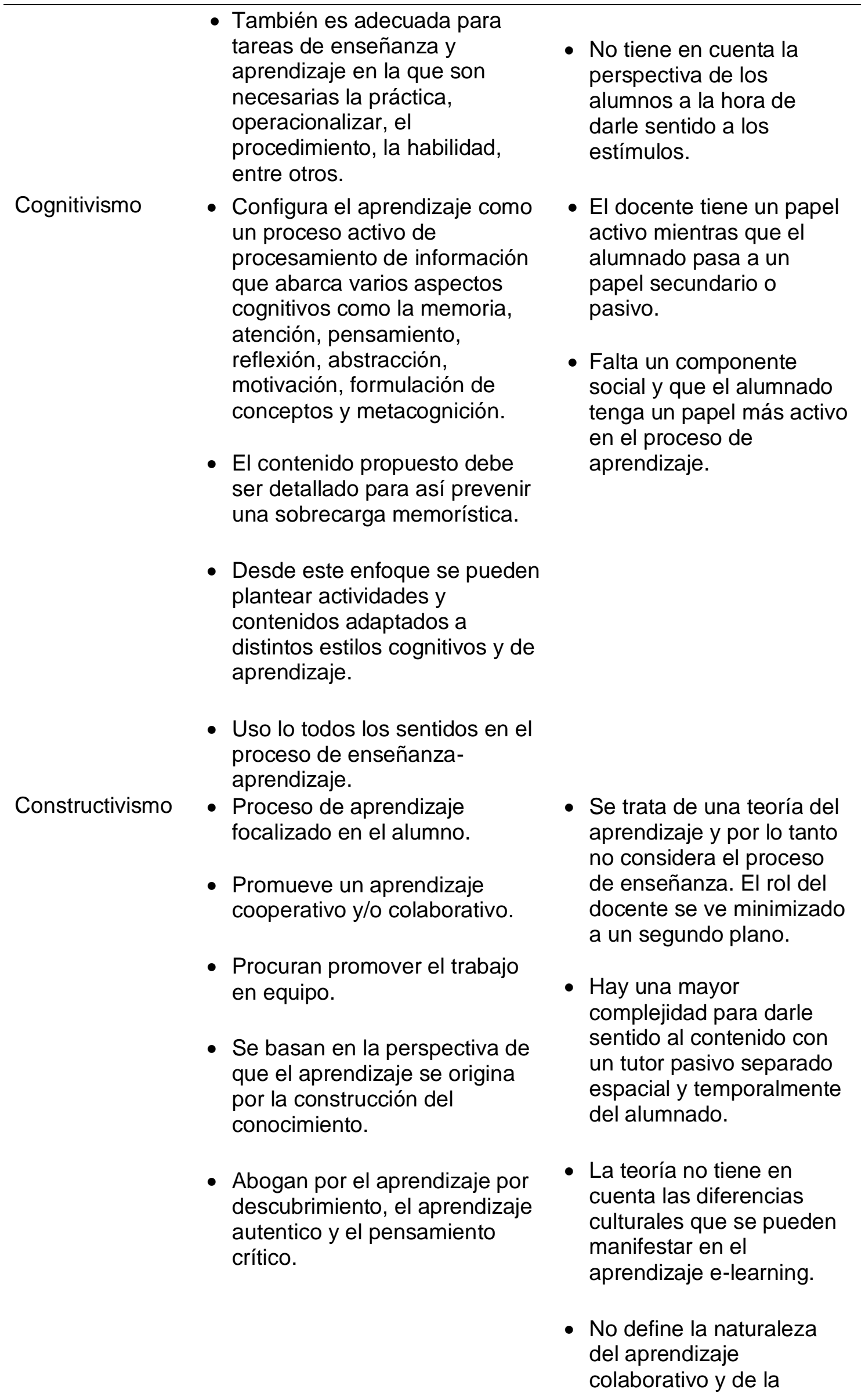


interactividad de los comportamientos en el elearning.

Conectivismo

- Se focaliza en el contexto social del alumnado y promueve un aprendizaje situado y un aprendizaje autentico.

- Se centra en la conectividad de los alumnos mediante el uso de la tecnología de la información y la comunicación para crear un aprendizaje por medio de redes y comunidades.

- También hace hincapié en la organización del conocimiento a través de la red de estudiantes.
- Hay una mayor complejidad para darle sentido al contenido con un tutor pasivo separado espacial y temporalmente del alumnado.

- El rol del tutor no está del todo bien definido.

- La naturaleza de los comportamientos colaborativos no está definida.

- El uso y el papel de las TIC no tiene un fundamento teórico.

\section{Ventajas y desventajas de la modalidad e-learning}

La modalidad e-learning, por sus características, presenta una serie de ventajas e inconvenientes con respecto a una metodología más tradicional. (Rosenberg, 2001)

Por un lado, sus beneficios serían:

- Ve reducido su coste, ya que no necesita invertir en traslado a la institución, materiales didácticos, alojamiento cerca de la institución en el que se impartiera en el caso de ser necesario, entre otros.

- La comunicación se ve agilizada debido al uso de internet.

- Los alumnos tienen cierta flexibilidad para acceder a los cursos siempre que tengan conexión a internet.

- Otra ventaja que presenta de manera innata es el desarrollo de un aprendizaje permanente importante para ser capaz de reciclarnos y desempeñar un buen papel laboralmente hablando.

Por otro lado, sus inconvenientes engloban:

- Inicialmente necesita inversión monetaria en infraestructura.

- Depende totalmente de las Tic para que se pueda ejecutar adecuadamente.

- Las tasas de abandono son altas con respecto a otras modalidades.

- Esto puede ser debido por la ausencia de un componente motivacional en el diseño, o bien por la tendencia a una metodología más tradicional.

- Los alumnos pueden verse sobrepasados por exceso de información y/o contenido. 


\section{Gamificación}

Ramírez-Cogollor (2014), que define la gamificación como la aplicación de estrategias (dinámicas y mecánicas) de juegos en contextos no jugables, ajenos a los juegos, con el fin de que las personas adopten ciertos comportamientos. La gamificación se ha aplicado en multitud de contextos tales como la salud, la educación y el turismo (Xu et al., 2013). Ubicando su aplicación en el ámbito educativo, una definición más contextualizada en este ámbito sería la que proponen Foncubierta y Rodríguez (2014). Estos autores proponen la gamificación con una técnica que el profesor utiliza al diseñar una actividad, tarea o proceso de aprendizaje a través del uso de elementos del juego, como insignias, puntuación, límite de tiempo, y/o mecánicas, como retos, aspectos competitivos entre otros, para mejorar la experiencia percibida del proceso de enseñanza-aprendizaje y guiar o cambiar el comportamiento que adoptan los estudiantes en el aula.

Al efectuar la gamificación, los alumnos mostrarán distintas motivaciones al presentarles la actividad en cuestión. Para desarrollar un ambiente propicio y atractivo para los estudiantes, habrá que tener en cuenta todas las sensibilidades e intereses de los estudiantes (Kim, 2015).

En relación a los elementos de los que se compone la gamificación, según Contreras y Eguía (2016), serían los siguientes:

- La actividad: se plantea como el tipo de actividad que se quiere diseñar de cara a alcanzar el objetivo planteado. Debe estar totalmente adaptada al contexto y a los estudiantes a los que vaya a ser dirigida, teniendo en cuenta la demografía, habilidad, entorno, entre otros factores, para así conseguir que la gamificación tenga un efecto significativo.

- Contexto: la gamificación se ve condicionada por el entorno donde va a ser aplicada, variando así el tipo de técnicas utilizadas. Concretamente hay que tener en cuenta, por un lado, el contexto organizacional, donde el docente podrá tener más o menos control y capacidad para gamificar. $\mathrm{Y}$, por otro lado, el contexto temporal, para ir adaptado la aplicación de esta estrategia con el paso del tiempo.

- Competencias y habilidades: cada persona es única, teniendo en cuenta sus virtudes y limitaciones. Por lo tanto, es necesario diseñar las actividades teniendo en cuenta las habilidades y competencias de los alumnos, intentando conseguir la inmersión del grupo en la actividad que le plantee.

- Gestión y supervisión: En relación con el punto anterior, el docente debe de estar capacitado para liderar dentro del aula a la hora de aplicar este tipo de estrategias. Hay varios aspectos que se deben tener en cuenta a la hora de aplicar la gamificación. Por un lado, es importante definir e implantar adecuadamente, teniendo en cuenta el momento en el que se aplica, ya que, si el docente tiene un rol muy definido, un cambio así puede hacer que los estudiantes se centren más en dicho cambio que en la actividad que plantea. Deberá haber un responsable que se encargue de supervisar la aplicación, y, posteriormente a su aplicación se deben revisar los resultados de la misma y proponer una serie de mejoras de cara a su posterior aplicación.

- Las mecánicas y elementos del juego: Una vez se cumplan todos los pasos anteriores, habrá que establecer los mecanismos y elementos del juego que se utilicen para conseguir el objetivo de la propia gamificación; motivar a los estudiantes para conseguir un proceso de enseñanza-aprendizaje donde los alumnos se diviertan a la vez que aprender. 
En este sentido los autores Werbach y Hunter (2015), se centraron en este último elemento llegando a diseñar y plantean la utilización del modelo Dinámica, Mecánica y Componentes (DMC), como la estrategia más apropiada al momento de diseñar la Gamificación. Este modelo se compone, piramidalmente de las siguientes dimensiones:

- Dinámica, estructura general o elementos que provocan la inmersión del estudiante promoviendo la participación activa, emociones, sentido de progresión y de mejora, entre otros.

- Mecánica, corresponde a las reglas y restricciones que puede poner un juego. Incluye los retos, cooperación, competición, feedback, adquisición de recursos, recompensas, transacciones, turnos, bonificadores/potenciadores, entre otros.

- Componentes, son los elementos que implementan y que se extrapolan de las mecánicas y dinámicas. Dentro de esta dimensión se sitúan los logros, avatares, coleccionables, peleas contra el jefe final, objetos utilizables, easter eggs, contenidos desbloqueables, regalos, rankings, niveles, puntos, misiones, equipos, bienes virtuales, entre otros.

\section{Principales Variables De La Gamificación}

Hay una serie de factores que se ven afectadas por la gamificación. En primer lugar, hay un estado psicológico relación con el juego que, según Csikszentmihalyi (2010), lo denomina flow (fluir). El estado mental relacionado con el flow, se caracteriza por la concentración enfocada y el mayor disfrute durante el desarrollo de una actividad (Shernoff, et al., 2003). Para Csikszentmihalyi (2010), el flow se relaciona con el aprendizaje, el desarrollo de talento, rendimiento académico y el logro creativo. En el ámbito de gamificación y aprendizaje basado en juego, los estudios señalan el aprendizaje y el currículo gamificado, serán cada vez más utilizados de tal forma que se pueda aprovechar el compromiso y la fluidez de los estudiantes (Crisp, 2014). En segundo lugar, la motivación es un elemento muy relevante ya que puede servir de apoyo para cumplir con uno de los objetivos más relevantes que se puede tener en el ámbito educativo, es decir, que el alumnado tenga interés por el contenido, atendiendo e interaccionando de forma elevada con el material. (Hanus y Fox, 2015) Las investigaciones señalan que existen más efectos positivos que negativos de la gamificación sobre la motivación (Sailer, Hense, Mayr y Mandl, 2017). Concretamente, se ha demostrado, que, a través de la gamificación, hay algunos elementos que bien implementados son capaces de aumentar la motivación de los estudiantes. Si se le plantean problemas o metas complejas los estudiantes tendrán un aumento de su motivación intrínseca, aumentando así su atención por la tarea. (Hamari et al., 2016) Aunque, hay algunas investigaciones que indican que, algunos elementos de la gamificación pueden llegar a dañar la motivación del estudiante. Por otro lado, según Landers, Bauer y Callan (2017), las personas deben comprometerse con el objetivo que persiguen para que este sea más motivador, lo anterior facilita el logro de las metas, aunque estos no sean tan realistas. Asimismo, según Lee y Hammer (2011), los juegos normalmente permiten al jugador reiniciar o jugar de nuevo, cometiendo errores recuperables. Esta libertad de fallar permite a los estudiantes experimentar sin miedo y aumenta el compromiso de los estudiantes y, en consecuencia, su satisfacción durante el proceso.

\section{Beneficios e inconvenientes de la gamificación}

Entre los múltiples aspectos positivos que proporciona indirectamente la gamificación, cabe destacar las aportaciones de (Sánchez Rivas y Pareja, 2015; Pisabarro y Vivaracho, 2018): 
- La gamificación hace que las actividades y el contenido sea más llamativo y aumenta la motivación en su aprendizaje mediante el logro de objetivos y reconocimientos.

- Las personas tienen una predisposición a jugar porque les proporciona una sensación placentera. Por este motivo la gamificación nos ayuda a enseñar y aprender mucho más mientras nos divertimos.

- La gamificación ayuda a que el estudiante sea más autónomo para poder realizar con seguridad las diferentes actividades que se proponga. Así el alumno siente que puede tomar sus propias decisiones y es responsable de sus consecuencias al dirigir su proceso. Esto implica que el alumno pueda decidir qué ritmo de aprendizaje llevar y por tanto desarrolle una serie de habilidades y destrezas por su cuenta.

- El trabajo en equipo es fundamental en la gamificación para que, de manera colaborativa, puedan conseguir las metas. Los juegos favorecen la socialización, en el caso de ser diseñados para participar en equipos.

- Se mantiene una retroalimentación constante y continua con los alumnos para que puedan conocer su progreso y su nivel de avance real. De esta manera se ayuda a que estos puedan realizar las correcciones necesarias para poder mejorar su competencia.

- En la gamificación los jugadores pueden ver en cualquier momento sus resultados, facilitando así la opción de poder comentar el progreso y obtener un refuerzo dentro de la actividad para que los docentes ayuden a que los jugadores no abandonen el juego frente a posibles problemas que puedan presentarse. De este modo se enseña a que fallar no es malo, y que gracias al ensayo-error pueden desarrollar la habilidad resolutiva de dichos problemas.

\section{Limitaciones e Inconvenientes de la gamificación}

Cabe destacar, que en el proceso de gamificación existen limitaciones e inconvenientes que dificultan su realización, principalmente (Sánchez Rivas y Pareja, 2015; Pisabarro y Vivaracho, 2018):

- La gamificación en el aula es un proceso complicado, por lo que hay que tener en cuenta la influencia de una alta variabilidad de factores, tales como la motivación, los conceptos, las actividades y que éstos respondan a competencias y habilidades, establece un desafío crucial al momento de realizar un diseño, es decir, que cumplan con los objetivos que se proponen.

- Usar la gamificación implica reducir y clasificar los contenidos de tal forma que se puedan relacionar con las actividades que se tienen propuestas. Lo anterior, exige la necesidad de priorizar temáticas de tal forma que se pueda concretar el proceso de gamificación.

- Elaborar materiales y recursos gamificados necesita de una gran cantidad de tiempo para diseñar, construir y desarrollar dichos materiales y actividades. Es por eso que los docentes, no pueden dedicarle tanto tiempo a este proceso puesto que tienen otras funciones que cumplir que también son útiles para su labor profesional. Al igual que el desarrollo de este tipo de estrategias lleva una mayor cantidad de tiempo, también tiene un coste monetario adicional, ya sea porque los realizas tú mismo como docente o porque hay que suscribirse a herramientas que den este tipo de servicios. Esto implica una renovación de los recursos de centros presenciales, además de una formación al equipo docente para ser capaz de elaborar o llevar a cabo esta estrategia pedagógica.

- Es necesario plantear un diseño adecuado para que los alumnos no olviden que su principal objetivo sigue siendo aprender y desarrollar los contenidos, teniendo en cuenta que, si le das demasiada importancia al juego pueden perder de vista 
este objetivo y centrarse solo en el juego en sí mismo. En relación con esto, es un inconveniente que puede aplicarse para las recompensas. Los alumnos pueden centrarse en solo obtener la recompensa sin interesarse por el hecho de aprender. Además, si les resulta difícil conseguir el objetivo para llegar a esa recompensa, pueden perder la motivación y rendirse pronto. Por consiguiente, se debe considerar que puede provocar desmotivación en los alumnos que queden en posiciones inferiores si no tienen recompensa. Por este motivo es importante focalizar el objetivo con recompensas intrínsecas para todos, significativas para los alumnos y, si puede ser, inesperadas.

- En contextos ajenos al ámbito educativo las personas que se vuelven adictos al juego, pueden desarrollar un aislamiento social, debido a que, se puede observar en que los videojuegos y la tecnología, en general, cuando tiene un enfoque demasiado individualizado se puede acabar adquiriendo una conducta antisocial. Para evitarlo, es importante tener en cuenta que se diseñen las actividades más enfocadas a trabajar en grupo que de manera individual.

A través de anteriores estudios se ha mostrado que, si bien la gamificación tiene aspectos muy positivos, aun no queda claro que aporte más beneficios que inconvenientes al implantarla (Koivisto y Hamari, 2019), por lo que se plantea este análisis cienciométrico para valorar la tendencia actual a través de la modalidad elearning.

\section{Objetivos o hipótesis}

A partir de la información expuesta en apartados anteriores se presentan los objetivos propuestos ante la realización del análisis cienciométrico.

\section{Objetivo general}

Analizar una serie temporal de producciones científicas indexadas en la base de datos Web of Science (WOS) relacionadas con la gamificación y el e-learning, publicadas en el periodo de 2014 a 2020.

\section{Objetivos específicos}

Los objetivos específicos propuestos para el análisis cienciométrico son los siguientes:

- Establecer el nivel de producciones científicas publicadas en las etapas contempladas en el periodo de 2014 a 2020.

- Determinar las principales instituciones y departamentos que publican producciones relacionadas con la gamificación y el e-learning

- Exponer la productividad de los principales autores indexados en WOS.

- Indicar los autores y artículos con mayor impacto, a través de su grado de citaciones, y esclareciendo el género del autor/a.

\section{Método}

El método cienciométrico es una disciplina científica, por la cual se pueden realizar análisis a través de una serie de indicadores que evalúan el progreso y estado científico de un tema en concreto (Fernández-Cano y Bueno, 1999; Padial Suárez y Fernández Cano, 2019), por lo que puede ser una alternativa de utilidad para valorar el estado de la cuestión y ajustarla a la propuesta que se pretende formular. (Expósito-López y Olmedo-Moreno, 2020). 
La gamificación es una pedagogía emergente por lo que tal como se indica en el marco teórico, no hay una combinación estandarizada de elementos de la gamificación que garantice la posibilidad de aprovechar todos sus beneficios. Por lo tanto, es importante evaluar la tendencia de las investigaciones realizadas para contextualizar una línea de actuación que garantice un uso adecuado de la gamificación en plataformas e-learning. (Berrocal-De luna y Megías-Ruiz, 2015)

El objeto de este análisis serían una serie de producciones científicas publicadas desde 2014 a la actualidad indexadas en la base de datos Web of Science (WOS), relacionadas con la gamificación y el e-learning en el ámbito educativo. Para realizar dicho análisis se tendrán en cuenta las producciones científicas publicadas por años y las distintas producciones publicadas diacrónicamente a nivel general y según el género; la producción por instituciones y departamentos; los autores más productivos y con mayor cantidad de citaciones; y un análisis de las principales coproducciones.

\section{La muestra/población}

La muestra obtenida a través del proceso de búsqueda realizado en la base de datos ha dado lugar a una matriz conformada por 228 producciones científicas, de las cuales 8 han sido eliminadas por falta de información para poder llevar a cabo el análisis y, posteriormente otros 10 artículos fueron eliminados de la muestra para poder aplicar el análisis a través de biblioshiny; por lo que la muestra está compuesta por 210 producciones científicas que tratan sobre la gamificación y la modalidad e-learning.

\section{Procedimiento}

El proceso de búsqueda y recogida de datos en la base de datos Web of Science (WOS) consistió en realizar una búsqueda avanzada a través de una serie de términos y/o conceptos clave que se utilizaron para componer la muestra, usándolos a través de distintas combinaciones de búsqueda durante el proceso. Los descriptores utilizados fueron los siguientes:

- Gamification

- Motivation

- E-learning

- Education

- Online

- MOOC

Todos los descriptores fueron utilizados, en distintas combinaciones, buscándolos en el título, resumen, y palabras clave de las producciones científicas comprendidas entre 2014 y 2020.

La técnica de muestreo utilizada para obtener la población necesaria para llevar a cabo la investigación es la de un muestreo no probabilístico, de carácter censal y de tipo intencionado o deliberado (McMillan y Schumacher, 2005) que a su vez ha sido validado por expertos.

Toda la información recogida por medio del proceso de búsqueda en la base de datos WOS fue organizada en una matriz de datos usando el programa Rstudio a través de la herramienta bibliometrix que permite hacer matrices y realizar el análisis cienciométrico considerando todas las variables contempladas al extraer la información de la base de datos WOS. Asimismo, para analizar los datos agrupados en la matriz realizada con bibliometrix se utilizó el programa Microsoft Excel 2016, ya que permite analizar grandes 
cantidades de datos, tal y como plantea la investigación. Sin embargo, teniendo en cuenta las limitaciones del programa, para ciertos análisis se utilizó el programa Statistical Package for the Social Sciences (SPSS) que permite obtener ciertos datos con más facilidad en comparación a Excel.

\section{Resultados}

A partir de este capítulo, se expone el análisis general a través de distintos aspectos, ya sea, diacrónicamente o a nivel de productividad, de la información extraída de las producciones científicas sobre el e-learning y la gamificación, publicadas indexadas a WOS y publicadas en el periodo comprendido entre 2014 a 2020.

\section{Serie temporal de artículos selecciona dos.}

En este apartado se analiza la distribución de las publicaciones de la temática escogida en el periodo establecido (2014-2020). Partiendo de la Ley de productividad de Price se espera que, siendo de un campo de estudio emergente, inicialmente haya un crecimiento lineal y, que con el paso del tiempo, alcanzara un crecimiento exponencial.

Así se plasma la serie temporal de las producciones científicas sobre el e-learning y la gamificación, extraídas de la base de datos WOS publicadas entre 2014 y 2020 (Figura 1 y Tabla 2). En primer lugar, en la siguiente tabla se muestra la cantidad de artículos publicados cada año y el porcentaje correspondiente a cada año de publicación.

Tabla 2

Número total de producciones por año

\begin{tabular}{lll}
\hline Año & Producciones & Porcentaje \\
\hline 2014 & 1 & $0,47 \%$ \\
2015 & 22 & $10,47 \%$ \\
2016 & 34 & $16,2 \%$ \\
2017 & 37 & $17,62 \%$ \\
2018 & 42 & $20 \%$ \\
2019 & 30 & $14,29 \%$ \\
2020 & 44 & $20,95 \%$ \\
\hline Total & 210 & $100 \%$ \\
\hline
\end{tabular}

Teniendo en cuenta los datos analizados en la tabla presentada se puede observar que la productividad va aumentado año tras año, si bien es cierto que en 2019 hay una caída de las publicaciones, en 2020 vuelve a aumentar el crecimiento de la productividad. Hay que mencionar que hay un crecimiento muy elevado desde 2014 a 2016, pasando de 1 publicación en 2014, a 22 publicaciones en 2015, hasta 34 publicaciones en 2016, observando un crecimiento exponencial desde el principio. Además, se expone un diagrama lineal de la producción diacrónica de las producciones científicas publicadas, para complementar los datos analizados en la tabla. 


\section{Figura 1}

Diagrama de la producción de artículos científicos en relación a la modalidad e-learning y la gamificación publicados entre 2014-2020.

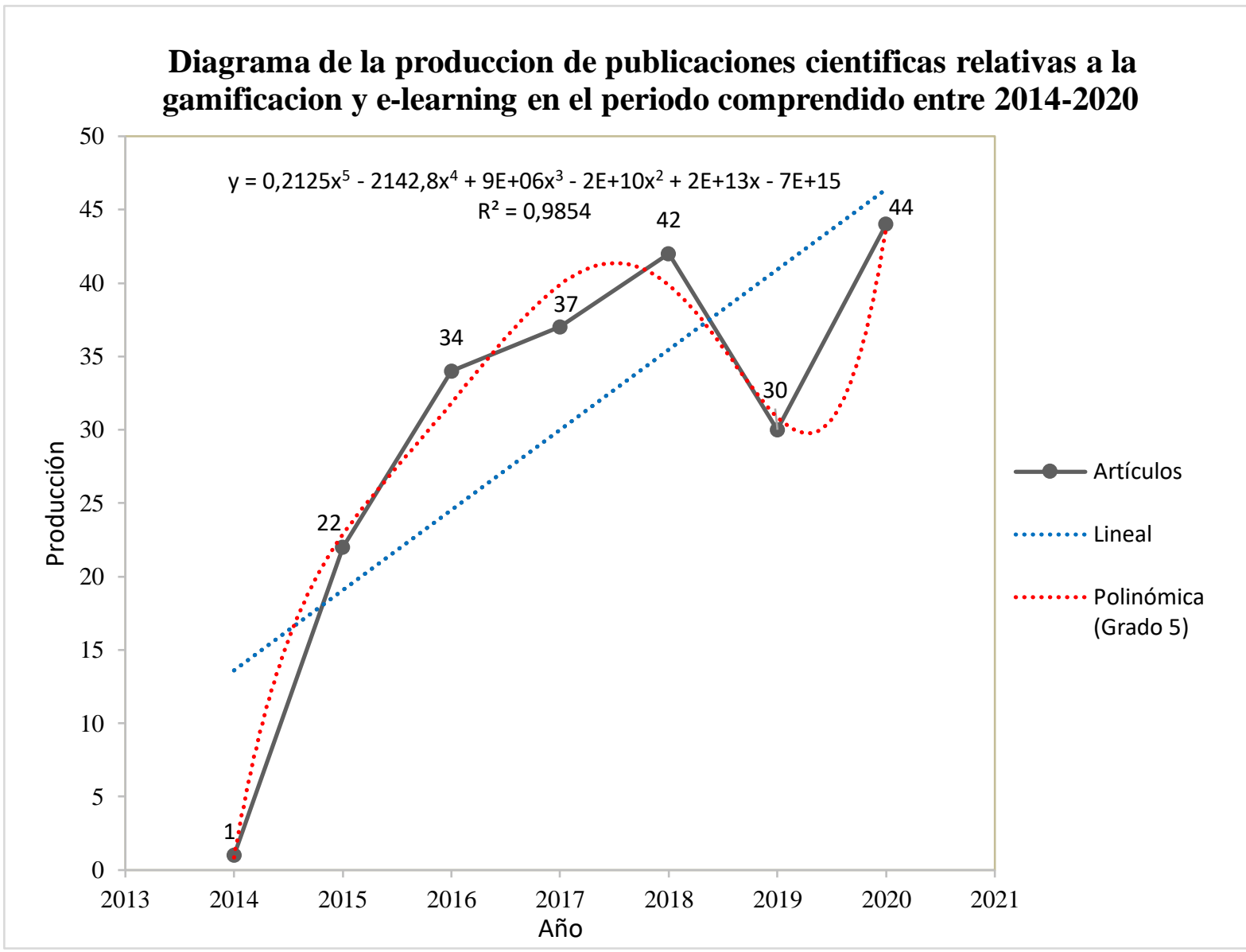

Por otro lado, también se incluyen el coeficiente de determinación $\left(R^{2}\right)$ y el coeficiente de correlación de Pearson $(r)$ y las líneas de tendencia lineal y polinómica para integrar más información y entender mejor los datos.

En este sentido, la línea de tendencia corresponde con la producción de artículos sobre e-learning y gamificación a través de la ordenación de tipo polinómica grado 5 (línea discontinua roja), la cual presenta un coeficiente de determinación $R^{2}=0,985$, y un coeficiente de correlación de Pearson $r=0,799$ (en sig. 0,05). Estos datos nos indican que existe una correlación positiva muy alta, debido a que está muy próxima a 1 , por lo que se extrapola que la publicación de los artículos ha ido aumentando exponencialmente, si bien en 2019 hay una caída, en 2020 vuelve a recuperar la tendencia exponencial. Por lo que, se espera, que la tendencia en los próximos años sea un aumento de la producción de artículos en relación a la temática de la investigación.

Conjuntamente, a través del diagrama y las líneas de tendencia, se pueden establecer 3 etapas de crecimiento y decrecimiento:

- De 2014 a 2018 se observa un crecimiento exponencial de la producción.

- En 2019 se observa un decrecimiento de las publicaciones.

- En 2020 vuelve a crecer el ritmo de producción, superando al pico más alto de productividad de 2018, volviendo a niveles exponenciales. 


\section{Producción institucional}

En este apartado se presentan las universidades más productivas en la publicación de producciones científicas relacionadas con el tópico de interés de la investigación. Por ello, se muestra la siguiente tabla con las instituciones más productivas, ordenadas de manera descendente, presentando primero las más productivas en artículos sobre elearning y gamificación.

\section{Tabla 3}

Producción de las principales Universidades

\begin{tabular}{|c|c|}
\hline Instituciones/Universidad & Publicaciones \\
\hline Salamanca & 7 \\
\hline Valladolid & 7 \\
\hline Georgia & 6 \\
\hline Murcia & 6 \\
\hline Finlandia oriental & 5 \\
\hline Hong Kong & 5 \\
\hline Universidad China Hong Kong & 4 \\
\hline Países Bajos & 4 \\
\hline Estado de Pennsylvania & 4 \\
\hline $\begin{array}{l}\text { Universidad de Ciencias Médicas de } \\
\text { Shiraz }\end{array}$ & 4 \\
\hline Alcalá de Henares & 4 \\
\hline Universidad Europea de Valencia & 4 \\
\hline Hospital Universitario de Ginebra & 4 \\
\hline Génova & 4 \\
\hline La Laguna & 4 \\
\hline Universidad Politécnica de Madrid & 4 \\
\hline Yale & 4 \\
\hline Otras universidades & 130 \\
\hline Total & 210 \\
\hline
\end{tabular}

A lo largo de la tabla se pueden observar las principales universidades productoras de artículos relacionados con el e-learning y la gamificación publicados entre 2014 y 2020. Se han enumerado las más destacables, estableciendo un corte de 4 publicaciones por institución. Así, se pueden identificar 5 agrupaciones bien diferenciadas en función de su productividad: 
- En primer lugar, estarían la Universidad de Salamanca y de Valladolid con 7 producciones científicas cada una.

- En segundo lugar, se situarían la Universidad de Murcia y de Georgia con 6 producciones científicas publicadas en ambos casos.

- En tercer lugar, se encontrarían la Universidad de Finlandia y la de Hong Kong, habiendo publicado 5 producciones cada una sobre la temática.

- En cuarto lugar, se localizarían un grupo de instituciones, principalmente españolas (Universidad Politécnica de Madrid, Alcalá de Henares, Universidad Europea de Valencia, entre otras) con 4 publicaciones sobre la temática de interés.

- En última instancia se encontrarían el resto de universidades, con una cantidad de publicaciones inferior a 4 artículos, siendo un total de 274 instituciones las que se hallarían por debajo de este umbral.

En relación a la información que proporciona la tabla, se presenta la siguiente figura, donde además de exponer las universidades más productoras:

\section{Figura 2}

Producción de las principales instituciones

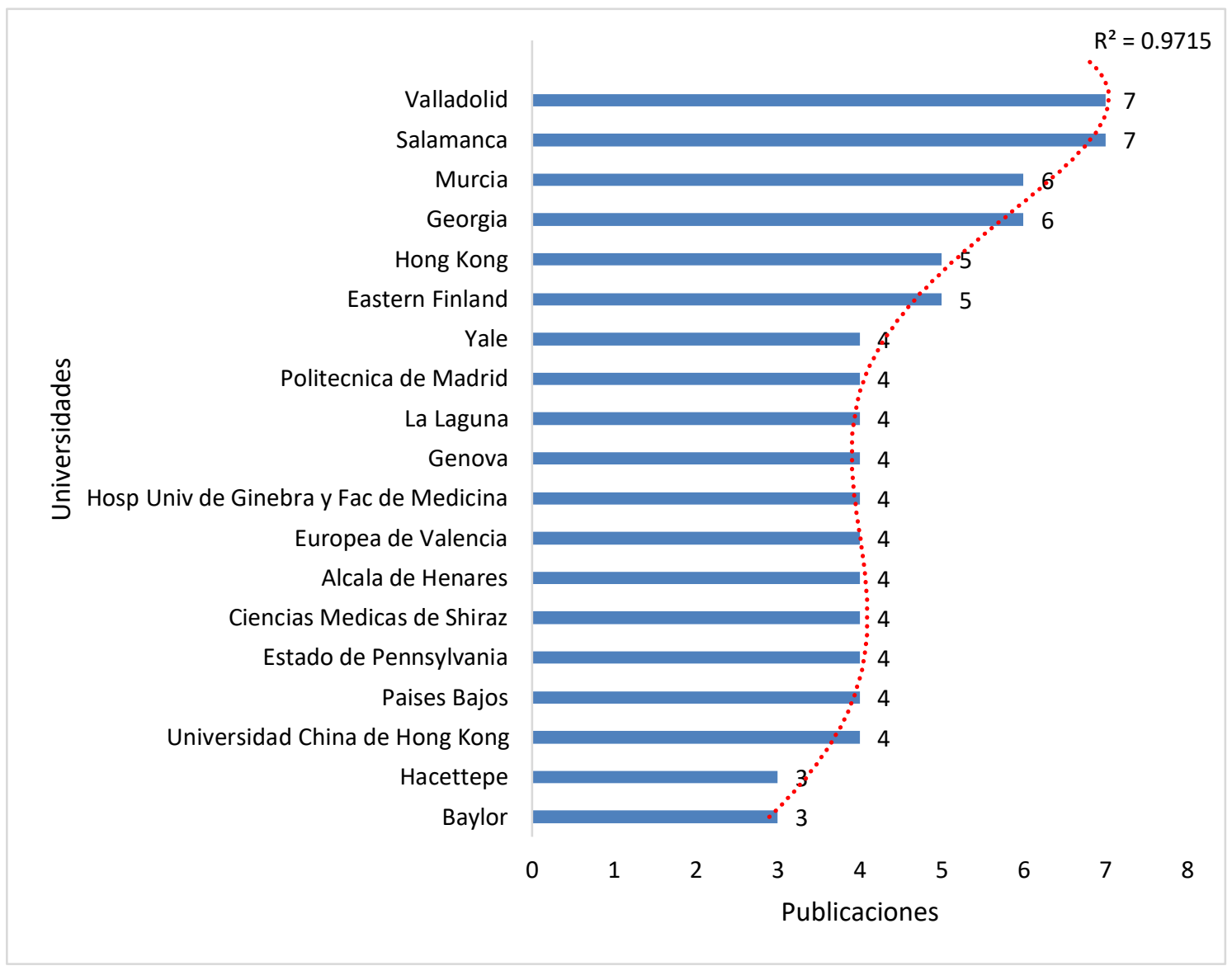

Además de los datos de las distintas universidades se presenta una línea de tendencia de tipo polinómica grado 6 , que implica un coeficiente de determinación $R^{2}=0,9715$ y, un coeficiente de correlación $r=-0,878(p<0,01)$. Por lo tanto, no se confirma la Ley de distribución de Lotka, teniendo en cuenta que no hay ninguna institución con una producción suficiente para ser una gran productora, y las universidades acaban distribuyéndose en un pequeño grupo de medianas productoras, habiendo publicado 
entre 2 y 7 artículos, y una gran mayoría de pequeñas productoras con una sola publicación.

\section{Producción de artículos por departamentos.}

Tras analizar las universidades más productivas, se pasa al análisis de los artículos producidos en función de su departamento o área temática de origen.

Cada universidad puede subdividirse en una diversidad de departamentos, y cada producción se asocia a un departamento u otro en función de la tendencia y la línea de investigación.

En la siguiente tabla quedan organizados los 220 artículos de la muestra original, repartidos en 10 departamentos, pertenecientes a las 291 instituciones que han publicado las producciones que forman parte de la muestra.

\section{Tabla 4}

Producción científica por departamentos o áreas temáticas

\begin{tabular}{|c|c|c|}
\hline Departamento & Producciones & Porcentaje \\
\hline $\begin{array}{ll}\text { Education } & \text { Educational } \\
\text { Research } & \end{array}$ & EER & 128 \\
\hline Computer Science & CS & 55 \\
\hline Engineering & EN & 27 \\
\hline Psychology & PS & 23 \\
\hline $\begin{array}{l}\text { Information Science Library } \\
\text { Science }\end{array}$ & ISLS & 7 \\
\hline $\begin{array}{l}\text { Science Technology other } \\
\text { topics }\end{array}$ & STOT & 7 \\
\hline Telecommunications & $\mathrm{TC}$ & 7 \\
\hline Social Sciences other topics & SSOT & 6 \\
\hline Business Economics & $\mathrm{BE}$ & 5 \\
\hline $\begin{array}{l}\text { Environmental } \\
\text { Ecology }\end{array}$ & ESE & 5 \\
\hline Total & 10 & $x$ \\
\hline
\end{tabular}

A partir de la tabla 4, se puede observar que la gran mayoría de las producciones pertenecen al ámbito de la educación, con un total de 128 producciones incluidas dentro del área de investigación Education Educational, seguida principalmente de Computer Science, Engineering y Psychology con 55, 27 y 23 producciones respectivamente.

\section{Autores más productivos.}

En esta sección se analizan los autores más productivos en función de la cantidad de publicaciones realizadas sobre la temática de investigación. En la figura 3 se puede observar en orden descendente los autores que más cantidad de producciones científicas han publicado sobre e-learning y gamificación. 
Figura 3

Autores más productivos

Autores más productivos

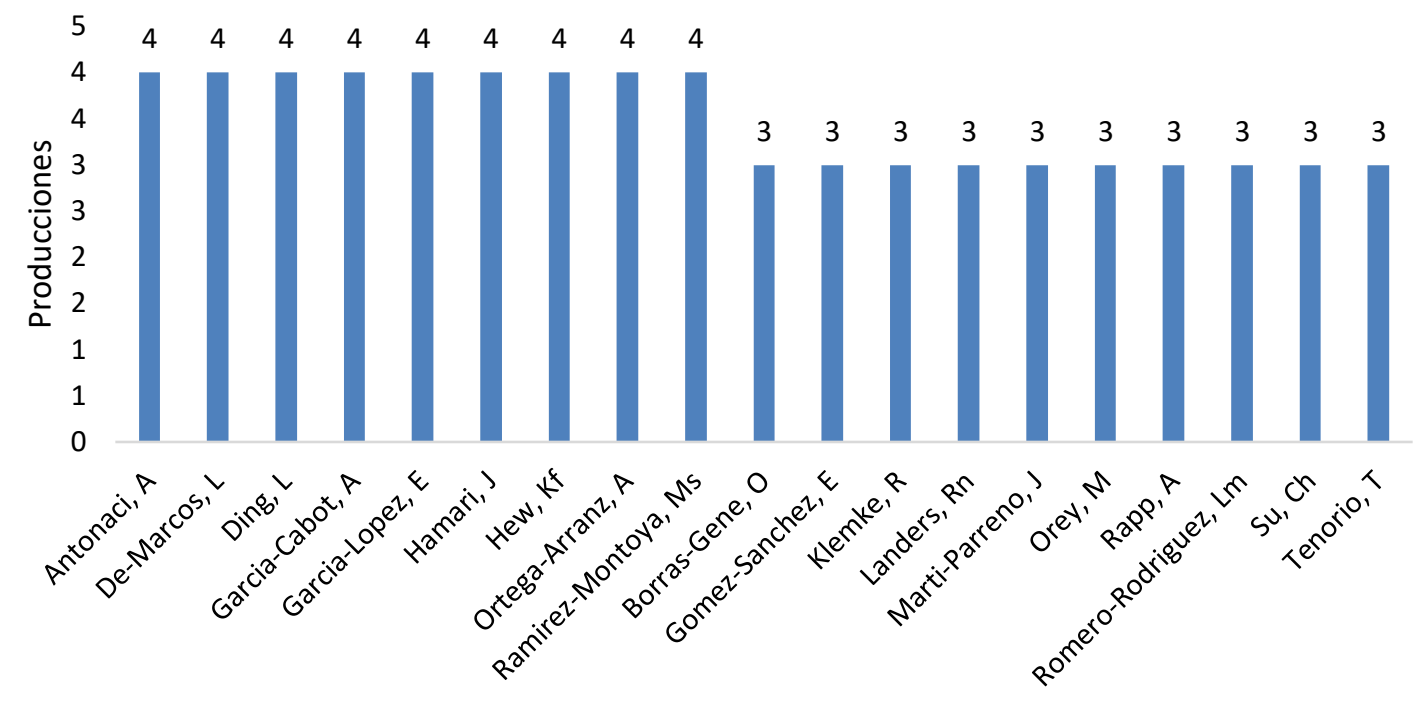

Autores

Se observa que los autores más productivos han llegado a publicar un total de 4 artículos, por lo que se puede deducir que es un área de estudio muy reciente y que aún puede abordarse en mayor profundidad.

TABLA 5

PRODUCTIVIDAD DE LOS AUTORES

AUTORES

ANTONACI, A

DE-MARCOS, L

DING, L

GARCÍA-CABOT, A

GARCÍA-LÓPEZ, E

HAMARI, J

HEW, KF

ORTEGA-ARRANZ, A

RAMÍREZ-MONTOYA, MS

BORRAS-GENE, $O$

GÓMEZ-SÁNCHEZ, E

KLEMKE, $R$

LANDERS, RN

Artículos

MARTI-PARRENO, J

OREY, M

RAPP, A

ROMERO-RODRÍGUEZ, LM

$\mathrm{SU}, \mathrm{CH}$

TENORIO, T

OTROS AUTORES

4

TOTAL

4

4

4

4

4

4

4

3

3

3

3

3

3

3

3

3

3

144

210 


\section{Distribución de los autores según su género}

En esta sección se va analizar la muestra de producciones científicas seleccionadas en relación al e-learning y la gamificación, publicados entre 2014 y 2020 según el género del autor. Se han tenido en cuenta tanto los autores principales como los coautores de las producciones científicas seleccionadas. En la figura 4 se presenta el total de autores divididos por género, presentando su total y su porcentaje equivalente.

\section{Figura 4}

Autores del fenómeno según su género

\section{Producciones cientificas según el género del autor}

Hombres

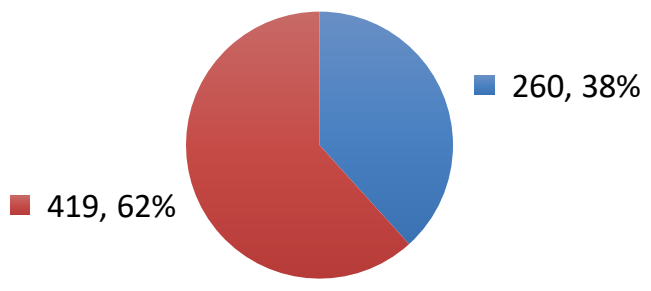

Tal y como se percibe en la figura, un $38 \%$ de los autores y coautores son mujeres y, en consecuencia, un $62 \%$ son hombres.

\section{Análisis de citas por autor}

En este caso se pone en valor los autores que más impacto han tenido en el ámbito del e-learning y la gamificación a través de la cantidad total de citaciones que han recibido. En la figura 5 se observa en orden descendente los autores con más citaciones en investigaciones relacionadas con e-learning y gamificación en el periodo de 2014 a 2020.

\section{Figura 5}

Autores con mayor impacto

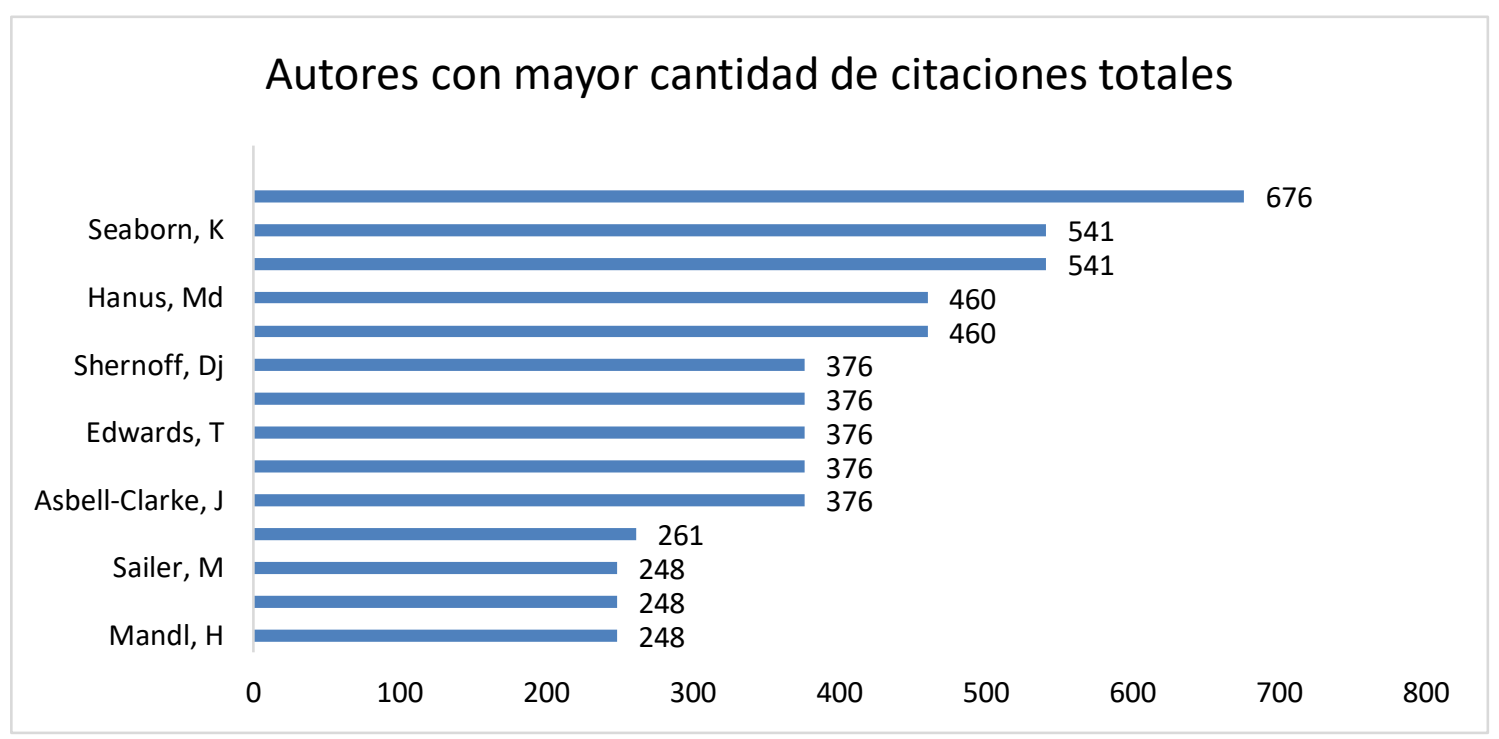


Como se puede observar en la figura autores como Hamari, Seaborn, Fels, Hanus y Fox son los autores con mayor impacto, teniendo $676,541,541,460$ y 460 respectivamente. Se trata de autores referenciados en esta investigación y que abogan por potenciar la motivación intrínseca a la hora de gamificar y tomar precauciones a la hora de diseñar una gamificación y no centrarse demasiado ni a largo plazo en elementos que promuevan una motivación extrínseca.

\section{Autores más citados desde un enfoque de género}

Al analizar los autores con mayor impacto dentro de la figura $X$ y evaluar los datos desde un enfoque de género, se observa que, de los 5 autores con mayor cantidad de citaciones, 3 de ellas son mujeres (Seaborn, Fels y Fox) y los 2 restantes hombres (Hamari y Hanus). Es un dato que contrasta con la cantidad de autores que han producido artículos sobre e-learning y gamificación en el periodo de 2014 a 2020 divididos por género, donde, como se ha indicado anteriormente, un $38 \%$ de los autores son mujeres y el $62 \%$ restante hombres.

\section{Artículos más citados}

Tras analizar los autores con mayor impacto, es importante evaluar las producciones científicas más relevantes y que más citaciones han recibido. A través de la siguiente figura, se pueden apreciar los artículos más citados publicados desde 2014 hasta 2020 en orden descendente:

\section{Figura 6}

Producciones científicas con mayor impacto

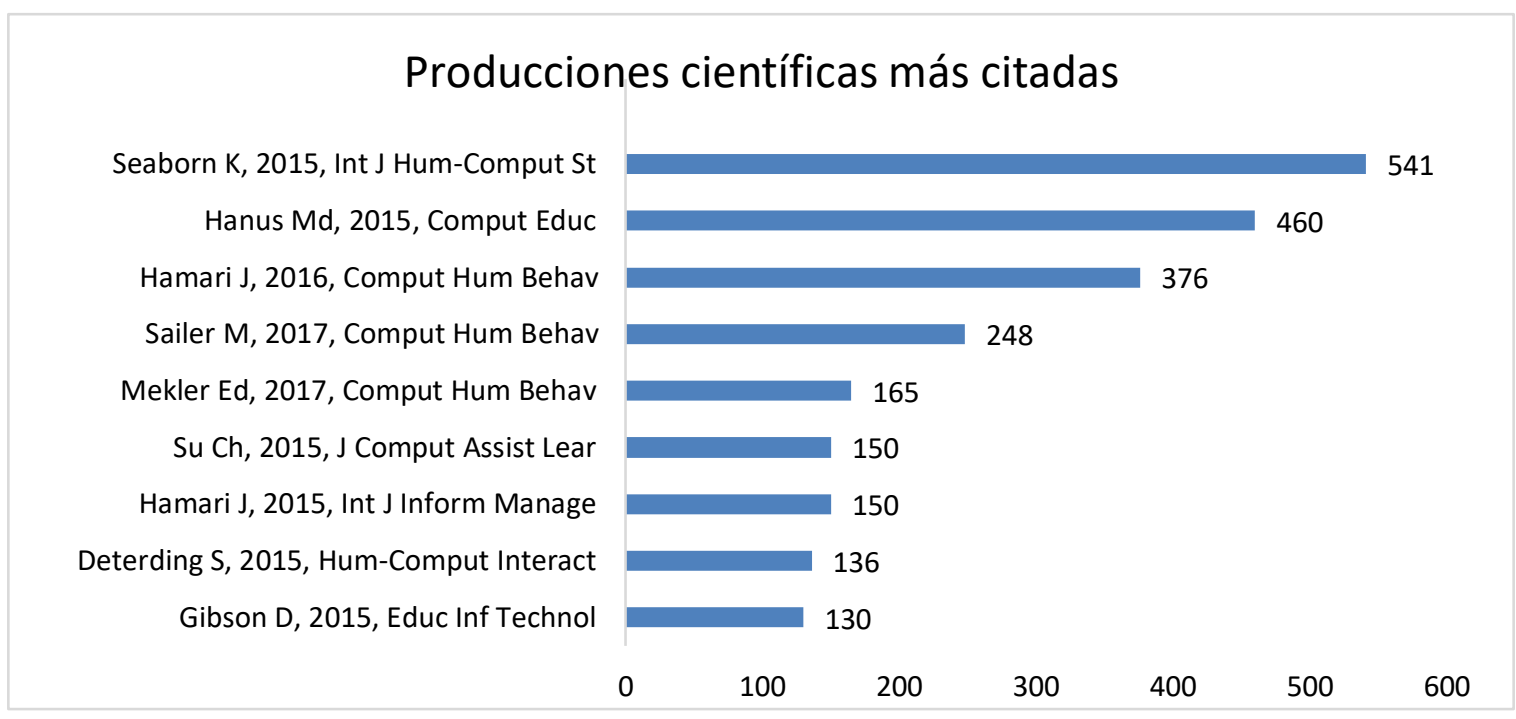

En general, en la figura 6 se puede apreciar que los datos concuerdan bastante con respecto al apartado anterior. Los artículos más relevantes tienen, generalmente, a los autores con mayor impacto.

\section{Mapa interactivo entre tres variables: Autor, institución, país}

Para finalizar, se evalúan 3 variables ya analizadas para ver como interactúan entre sí. Por lo que, en la figura 7, se muestra la interacción entre la nacionalidad, la universidad desde la que se publica y los autores relacionados con ésta. 


\section{Figura 7}

Interacción entre institución, autor y país

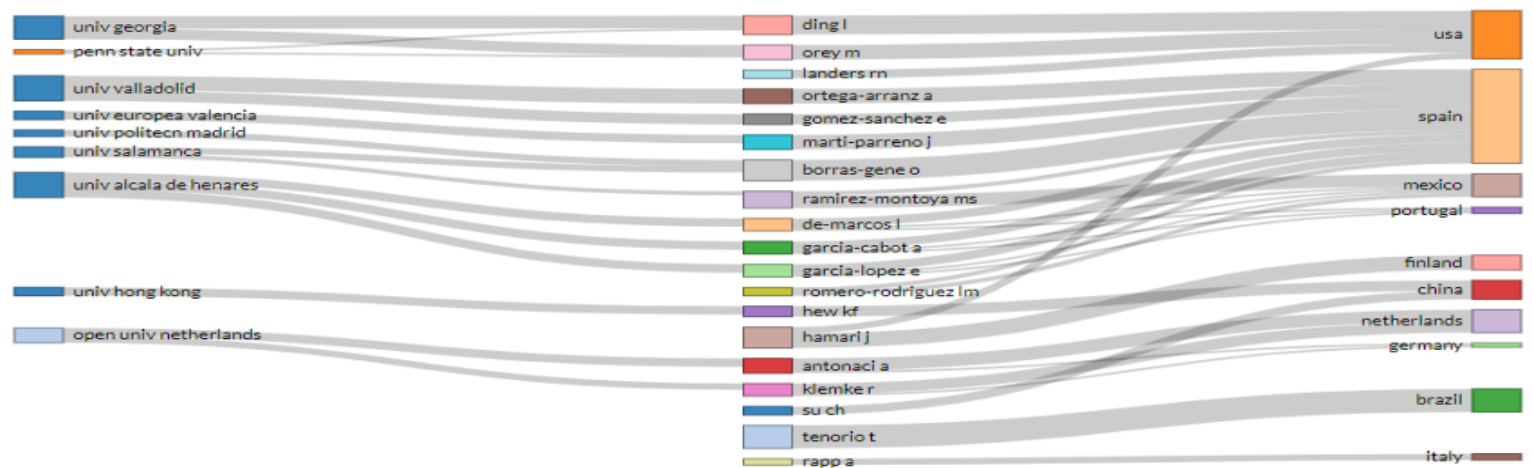

Según los datos de la figura 7 principalmente se han publicado más artículos por parte de autores de nacionalidad española y estadounidense, provenientes principalmente de la Universidad Alcalá de Henares y de Valladolid en el caso de España y de la Universidad de Georgia en el caso de EEUU.

\section{Discusión y conclusiones}

Una vez expuestos los datos y el análisis realizados sobre estos, se revela si se han cumplidos los objetivos fijados ante la elaboración del análisis cienciométrico, y los resultados más relevantes del mismo.

Con respecto a los objetivos planteados, se han completado todos y cada uno, haciendo un análisis de la temática en el periodo que comprende 2014 a 2020, confeccionando cada análisis especifico, centrado principalmente en evaluar autores, instituciones, productividad e impacto.

En primer lugar, en la serie temporal de producción, se confirma la ley de Price en la fase de crecimiento exponencial, y, según la línea tipo polinómica (grado 5) que se observa en la figura 1, la tendencia exponencial se espera que continua en los siguientes años de publicación.

En segundo lugar, a nivel institucional no se cumple la ley de distribución de Lotka, donde unas pocas Universidades concentran una mayor cantidad de publicaciones acerca de la temática, pero no llegan a ser grandes productoras, y la gran mayoría de instituciones han publicado solo un artículo sobre el fenómeno de estudio. Asimismo, a nivel de departamentos se observa que la mayoría de las producciones científicas estan enfocadas al ámbito de la educación directamente.

En tercer lugar, en la productividad de los autores se puede apreciar que no hay autores con un nivel muy alto de producción, teniendo 4 producciones los autores con una mayor cantidad de publicaciones.

Por otro lado, hablando del impacto de autores y artículos en específico, hay una tendencia clara. Los autores con un mayor impacto plantean una perspectiva en la que se respalda una gamificación que se centre en trabajar la motivación intrínseca y de no plantear unos elementos gamificados que se dirijan a la motivación extrínseca a largo plazo; en esta línea, las producciones científicas con mayor impacto principalmente coinciden con los autores que acumulan una mayor cantidad de citaciones. 
A nivel de género, si bien es cierto que existe una mayor cantidad de hombres publicando sobre el fenómeno en cuestión, al tener en cuenta los autores con mayor impacto, destaca que, entre los 5 autores con una mayor cantidad de citaciones acumuladas, y que presentan cierta distancia en su impacto con respecto a los demás autores, 3 son mujeres (Seaborn, Fels y Fox) y 2 hombres (Hamari y Hanus), invirtiendo así la tendencia general.

\section{Limitaciones y futuras líneas de investigación}

La principal limitación que presenta este estudio es la falta de información en algunas producciones indexadas en la base de datos WOS. Parte de las producciones científicas seleccionadas no presentaban cierta información, necesaria para poder llevar a cabo el análisis con la muestra completa a través de Biblioshiny, por lo que esta situación conlleva a la perdida de parte de la muestra, y por tanto de parte de los datos a la hora de realizar el análisis.

Por otro lado, otra limitación sería la falta de un análisis del contenido de la muestra para profundizar en la tendencia teórica y práctica de la aplicación de la gamificación en plataformas e-learning, lo cual puede ser una línea de investigación en el futuro, y comprobar si la tendencia del fenómeno corresponde con la que plantean los autores y artículos con mayor impacto.

De cara a futuras investigaciones, partiendo del análisis inicial planteado en este estudio, se pueden abrir muchas bifurcaciones, además de la ya mencionada con respecto a la tendencia actual del fenómeno, ya sea profundizar en la productividad e impacto generalizado de todos los autores que han publicado sobre objeto de estudio, desde una perspectiva de género; analizar las coproducciones entre autores y colaboraciones entre las distintas instituciones; indagar sobre la calidad de las producciones desde el impacto y cuartil de las fuentes de publicación, entre otras. De esta manera tendríamos un mayor conocimiento sobre el objeto de estudio para redirigir su investigación teórico-practica para que se establezca si realmente la gamificación es una pedagogía útil, en qué contextos y situaciones puede y debe aplicarse a nivel virtual, si realmente cubre las necesidades que exigen las plataformas e-learning y qué componentes, elementos y mecánicas son relevantes para que dicha pedagogía cumpla su función.

\section{Referencias}

Berrocal de Luna, E., \& Megías Ruiz, S. (2016). Indicadores de calidad para la evaluación de plataformas virtuales. Revista Internacional de Aprendizaje $\quad y \quad$ Cibersociedad, 19(2). https://doi.org/10.37467/gka-revciber.v19.870

Contreras, R. S., \& Eguía, J. L., Universitat Autònoma de Barcelona, \& Institut de la Comunicació. (2017). Experiencias de gamificación en las aulas. https://ddd.uab.cat/pub/llibres/2018/188188/ebook 15.pdf

Crisp, G. T. (2014). Assessment in next generation learning spaces. En K. Fraser (Ed.), International Perspectives on Higher Education Research (Vol. 12, pp. 85-100). Emerald Group Publishing Limited. https://doi.org/10.1108/S1479362820140000012009
Csikszentmihalyi, M. (2010). Fluir: Una psicología de la felicidad (N. López Buisán, Trad.; 1. ' ${ }^{a}$ ed.). Editorial Kairós.

Expósito-López, J., \& Olmedo-Moreno, E. M. (2020). Scientometric analysis of publications on guidance, mentoring, and tutorial action registered on databases. Formación universitaria, 13(3), 123$138 . \quad$ https://doi.org/10.4067/S071850062020000300123

Fernández-Cano, A. y Bueno, A. (1999). Synthesizing scientometric patterns in Spanish educational research. Scientometrics, 46(2), 349- 367

Foncubierta, J. M., \& Rodríguez, C. (2014). Didáctica de la gamificación en la clase de español. Madrid: Edinumen. https://www.edinumen.es/spanish_challenge/gamif icacion_didactica. 
García Aretio, L. (2020). Bosque semántico: ¿educación/enseñanza/aprendizaje a distancia, virtual, en línea, digital, eLearning...? RIED. Revista Iberoamericana de Educación a Distancia, 23(1).

https://doi.org/10.5944/ried.23.1.25495

Hamari, J., Shernoff, D. J., Rowe, E., Coller, B., AsbellClarke, J., \& Edwards, T. (2016). Challenging games help students learn: An empirical study on engagement, flow and immersion in game-based learning. Computers in Human Behavior, 54, 170179. https://doi.org/10.1016/j.chb.2015.07.045

Hanus, M. D., \& Fox, J. (2015). Assessing the effects of gamification in the classroom: A longitudinal study on intrinsic motivation, social comparison, satisfaction, effort, and academic performance. Computers \& Education, 80, 152161.

https://doi.org/10.1016/j.compedu.2014.08.019

Kibuku, R. N., \& Ochieng, D. O. (2018). Formulating an e-learning theory: A grounded theory approach. Proceedings of the Second African Conference for Human Computer Interaction: Thriving Communities, 1-5. https://doi.org/10.1145/3283458.3283492

Kim, B. (2015). Chapter 5. Designing gamification in the right way. Library Technology Reports, 51(2), 2935 .

Koivisto, J., \& Hamari, J. (2019). The rise of motivational information systems: A review of gamification research. International Journal of Information Management, 45, https://doi.org/10.1016/j.jijinfomgt.2018.10.013

Landers, R., Bauer, K., \& Callan, R. (2017). Gamification of task performance with leaderboards: A goal setting experiment. Computers In Human Behavior, 71, 508-515. https://doi.org/10.1016/j.chb.2015.08.008

Lee J. J, Hammer J (2011) Gamification in education: what, how, why bother? Academic Exchance Quarterly 15(2):1-5.

McMillan, J., y Schumacher, S. (2005). Investigación Educativa. Madrid: Pearson Educación.

Padial Suárez, J. J., \& Fernández Cano, A. (2019). Análisis cienciométrico de Tesis Doctorales Españolas sobre Altas Capacidades y $\begin{array}{lll}\text { sobredotación } & \text { (1986- } & \end{array}$ https://doi.org/10.30827/Digibug.57762

Pisabarro, A. M., \& Vivaracho, C., (2018). Gamificación en el aula: Gincana de programación. ReVisión: Revista Hispanoamericana de Educación Universitaria de la Informática, 11(1), 85-93.

Ramírez-Cogollor, J. L. (2014). Gamificación. Mecánicas de juegos en tu vida personal y profesional. Editorial SCLibro. Madrid, España.
Rodrigues, H., Almeida, F., Figueiredo, V., \& Lopes, S. L. (2019). Tracking e-learning through published papers: A systematic review. Computers \& Education, 136, 87-98.

Rosenberg, M. J. (2001). E-learning: Strategies for delivering knowledge in the digital age. McGrawHill.

Sailer, M., Hense, J., Mayr, S., \& Mandl, H. (2017). How gamification motivates: An experimental study of the effects of specific game design elements on psychological need satisfaction. Computers in Human Behavior, 69, 371-380. https://doi.org/10.1016/j.chb.2016.12.033

Sánchez-Rivas, E. y Pareja-Prieto, D. (2015). La gamificación como estrategia pedagógica en el contexto escolar. En Ruiz-Palmero, J., SánchezRodríguez, J. y Sánchez-Rivas, E. (Edit.). Innovaciones con tecnologías emergentes. Málaga: Universidad de Málaga.

Shernoff, D. J., Csikszentmihalyi, M., Shneider, B., \& Shernoff, E. S. (2003). Student engagement in high school classrooms from the perspective of flow theory. School Psychology Quarterly, 18(2), 158176. https://doi.org/10.1521/scpq.18.2.158.21860

Valverde-Berrocoso, J., Garrido-Arroyo, M., BurgosVidela, C., \& Morales-Cevallos, M. (2020). Trends in Educational Research about e-Learning: A Systematic Literature Review (2009-2018). Sustainability, $12(12), \quad 5153$. https://doi.org/10.3390/su12125153

Velazco Flórez, S. Y., Abuchar Porras, A., Castilla, I., \& Rivera, K. (2017). e-Learning: Rompiendo fronteras. Redes De Ingeniería, 91-100. https://doi.org/10.14483/2248762X.12480

Werbach, K., \& Hunter, D. (2015). The gamification toolkit: Dynamics, mechanics, and components for the win. Wharton Digital Press.

Xu, F., Weber, J., \& Buhalis, D. (2013). Gamification in tourism. En Z. Xiang \& I. Tussyadiah (Eds.), Information and Communication Technologies in Tourism 2014 (pp. 525-537). Springer International Publishing. https://doi.org/10.1007/978-3-319-03973-2_38 